\title{
Efficiency of Immunological Methods in the Diagnosis of Active Tuberculosis in Children
}

\author{
Anna Starshinova ${ }^{1}$, Irina Dovgaliuk ${ }^{1}$, Natalia Korneva ${ }^{1}$, Ulia Ovchinnikova ${ }^{1}$, Olga Yakunova ${ }^{1}$, Elena Potapenco ${ }^{1}$, \\ Maria Shulgina ${ }^{1}$, Elmira Zilber ${ }^{1,2}$ and Piotr Yablonskii ${ }^{1,2}$ \\ 1. Department of Phthisiopulmonology, St. Petersburg Research Institute of Phthisiopulmonology, St. Petersburg 191036, Russian
} Federation

2. Department of therapy, St. Petersburg State University, St. Petersburg 199034, Russian Federation

\begin{abstract}
Nowadays, no clear diagnostic criteria for tuberculosis in children exist as absolute majority of tuberculosis cases in childhood have no bacterial excretion, unlike adults where bacterial excretion is observed not rarely. This fact leads to necessity to implement immunological methods in diagnostic complex in children. Tuberculin test is a routine one which is used for diagnosis of tuberculosis. However, there are a number of factors which interfere with the diagnostic value of tuberculin test. This study helped to ascertain the superiority of DST (Diaskintest) and QFT-G (QuantiFERON®-TB Gold IT) with respect to the wealth of information they provide when compared with the TST (tuberculin screening test) in determining the activity of tuberculosis infection in children. The evaluation parameters of diagnostic value (DSS (diagnostic sensitivity), the DSC (diagnostic specificity), PPV (positive predictive value) and NPV (negative predictive value), and DE (diagnostic efficiency)) with DST, QFT and TST (tuberculin skin test) were: DSS-84.3\%, DSC-59.1\%, PPV-61.9\%, NPV-82.7\%, DE-71.7\%) with QFT (DSS-78.4\%, DSC-68.2\%, PPV-57.7\%, NPV-85.1\%, DE-73.3\%) were significantly higher than with TST (DSS-91.5\%, DSC-15.7\%, PPV—74.4\%, NPV—45.8\%, DE-53.6\%). New immunologic methods DST and QFT-G have higher specificity and diagnostic value in comparison with TST that makes their implementation in the diagnosis of TB in children essential.
\end{abstract}

Key words: Tuberculosis, children, Diaskintest, QuantiFERON-TB test, immunology.

\section{Introduction}

In Russian Federation, TILN (tuberculosis of intrathoracic lymph nodes) prevails in children, commonly found in $74 \%$ of all cases [1, 2]. Nowadays, no clear diagnostic criteria for tuberculosis in children exist as absolute majority of tuberculosis cases in childhood have no bacterial excretion unlike adults where bacterial excretion is observed in $36 \%$ cases of the disease [3, 4]. This fact leads to necessity to implement immunological methods in diagnostic complex in children.

Tuberculosis is diagnosed by a data set: clinical symptoms, positive TST (tuberculin skin test) and radiologic data. However, the frequent absence of the intoxication symptoms of tuberculosis in children

Corresponding author: Anna Starshinova, Ph.D., research field: pediatrics. E-mail: starshinova_777@mail.ru. along with the increasing comorbidity in modern scenarios significantly impedes the assessment of the intoxication symptoms and nature of sensitivity to tuberculin, which has a numeric response in $60 \%$ of the patients with tuberculosis disease [5, 6]. Often TST gives an incorrect result, because many factors affect diagnostic value of tuberculin test. BCG (Bacillus Calmette-Guerin) vaccination, growth of allergic disorders and concomitant diseases in children lead to false positive or sometimes to false negative results of the test $[7,8]$. Due to this, new immunologic methods and criteria are required for diagnosis of tuberculosis infection in children. This indicates late detection of the disease; therefore, there is an urgent need for the introduction of new immunological tests for early detection and determination of the activity of the tuberculosis infection. 
The aim of presenting research was to identify immunologic features in children with tuberculosis of intrathoracic lymph nodes.

\section{Material and Methods}

Between 2010 and 2013, a prospective study of the information by employing in a complex diagnosis of tuberculosis in children was conducted in the Department of Pediatric Phthisiopulmonology. We examined 213 children from 3 to 14 years old with a positive tuberculin test, of whom 102 (47.8\%) were between 3 to 6 years old and 111 (52.2\%) were between 7 to 14 years old. The patients were assessed by clinical symptoms and results of CT (computer tomography). Assessment of intrathoracic LNs (lymph nodes) in children using X-ray methods was performed in line with the recommendations, according to which the transverse dimension of the lymph nodes in children (from 3 to 14 years old) should not exceed 10 $\mathrm{cm}$ depending on the group and the age of the child; however, that did not exclude the presence of pathological changes, including specific changes, in the smaller nodes [9]. According to Lazoreva et al. [10], Gegeeva [11], and Dauletova [12], all LNs between $5 \mathrm{~mm}$ to $10 \mathrm{~mm}$ should be treated as manifestations of tuberculosis in intrathoracic LN, although, the standard CT imaging studies can be the limit for visualization of the LNs according to the criteria of "imaging".

According to the results, they were split in two groups. The first group includes 70 children who were infected by MBT (Mycobacterium tuberculosis), but have no tuberculosis disease-it was taken in the research as a control group; the second group includes 143 children with tuberculosis of intrathoracic lymph nodes. Both groups were tested by DST using recombinant tuberculosis allergen based on MBT specific proteins: ESAT-6 (early secreted antigenic target $6 \mathrm{kD}$ protein) and CFP-10 (culture filtrate protein 10 ), and by its nature it represents an immunologic skin-test. This test is designed in Russia and used for diagnosis of tuberculosis since 2009. An analysis of the results of the tuberculin skin test (administration of the purified tuberculin in standard dilution (ready form)) and DST (tests with recombinant TB allergens in standard dilution) was conducted [13, 14].

In addition QuantiFERON test ("Tubinferon” test system) has been used. Before performing these tests, a venous blood sample was drawn for QFT (QuantiFERON-TB test), which is the reference method and allows a qualitative assessment of the information contained. The QuantiFERON®-TB Gold In-Tube is a diagnostic tool designed for the diagnosis of tuberculosis in vitro. This method is based on using a peptide cocktail simulating ESAT-6, CFP-10 and TB7.7 (p4) proteins to stimulate the cells in heparinized whole blood. Determination of the quantification of IFN- $\gamma$ in anda-TB (BioChimMak) as performed to identify in vitro the cellular response to the stimulation of these peptide antigens associated with Mycobacterium tuberculosis infection. Since tuberculosis in literature described as the disease with immunosuppressive pattern, the following immunologic parameters were chosen for assessment: leucocytes' subsets identification $\left(\mathrm{CD}^{+}, \mathrm{CD}^{+}, \mathrm{CD}^{+}\right.$, $\mathrm{CD}^{+} / \mathrm{CD}^{+}, \mathrm{CD}^{+}, \mathrm{CD}_{16}{ }^{+}, \mathrm{CD}^{+}{ }^{+}, \mathrm{CD} 25^{+}, \mathrm{CD}^{+} 5^{+}$ and HLAII), induced cytokines levels (TNF-á, IL-2, IL-4 and INF- $\gamma$ ), and assessment of antibodies IgA, IgG and IgM (anda-tb ELISA) .

Apart from these X-ray method diagnosis ((MSCT (multislice spiral computed tomography) and MSCT-AG (MSCT angiography)) and X-ray examination were done using a spiral CT scanner with a multirow detector (multislice) “Aquilion-32” (Toshiba Medical Systems Corporation, Japan).

In clinical example 1 (Fig. 1), child P.S., 5 years old, was vaccinated by BCG. Tuberculin test in 2007-p 13 mm, 2008—p 15mm, 2009—p 16mm and 2010—p $15 \mathrm{~mm}$. In anamnesis, she has allergy. In examination, $\mathrm{CD}_{16}{ }^{+}$is low, DST-negative, QFT-negative, IgG is low, and no symptoms of intoxication. In CT, in lungs and intrathoracic lymph nodes tuberculosis changes are not identified. In this 


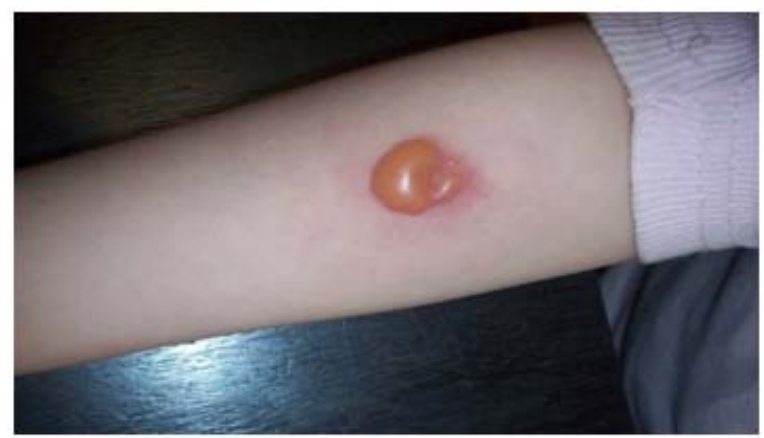

(a) Tuberculin test is positive

Fig. 1 Child P.S., 5 years old.

example, high result of TST is influenced by allergy growth.

In clinical example 2 (Fig. 2), child P.M. was 3 years old. She has a contact with her father, who is ill since 2008 with lung tuberculosis MBT (+) with has MDR (multidrug resistance). BCG vaccination, preventive course of therapy in 2007 and 2008. Tuberculin test: 2009-p 6mm, 2010-p 18mm; $\mathrm{CD}^{+} 6^{+}$is high, DST-p 20mm, QFT-positive, IgG is high, symptoms of intoxication. CT and CT-Ag-lymph nodes $>1.0 \mathrm{~cm}$ paratracheal, bifurcation, bronchopulmonal groups. In this example, child who had tuberculosis of intrathoracic lymph nodes and course of therapy has been administered.

All the data was processed employing the variation statistics methods using the software Microsoft Office Word Excel 2007, Statistica 8. The difference was considered reliable when $P<0.05$. The Mann-Whitney (U Test) was used to compare the differences between

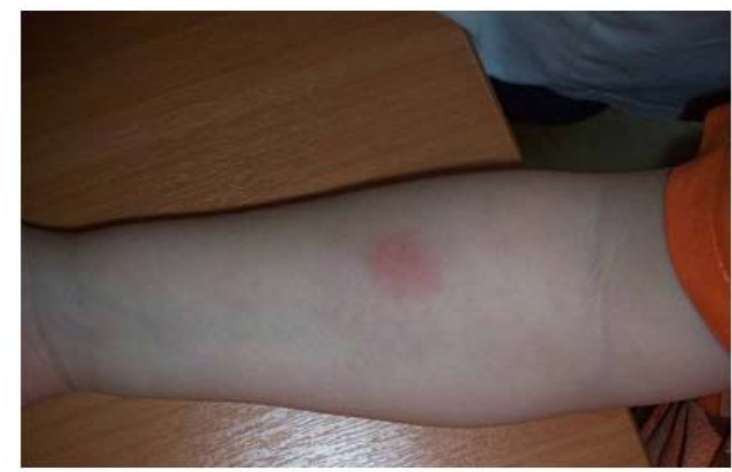

(a) Tuberculin test $-\mathrm{p} 18 \mathrm{~mm}$

Fig. 2 Child P.M., 3 years old.

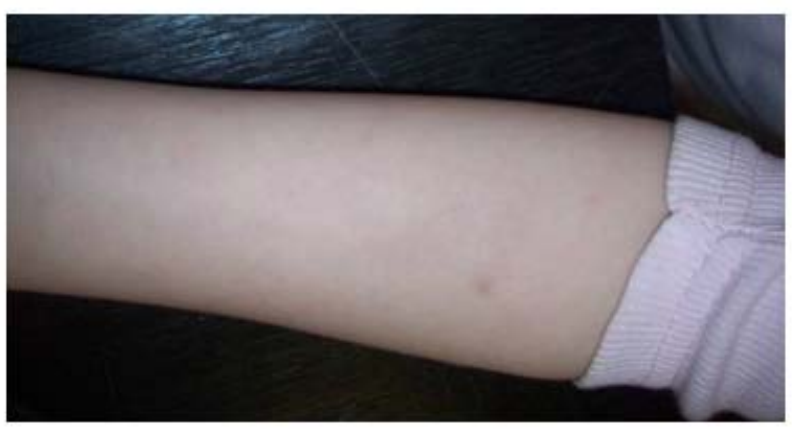

(b) Diaskintest $\mathbb{8}$ is negative

two independent groups (for nonparametric data). The mean $(M)$ and standard error of the mean $(M)$ were deduced. Pearson's Correlation Coefficient $(r)$ was used to determine the strength of the relationship between two continuous variables. $P$ value less than 0.05 was considered significant. Spearman's rank correlation coefficient was also used. The diagnostic accuracy of the tests employed was analyzed as well as the method used to calculate the operating characteristics: DSS, DSC, PPV, NPV and DE.

\section{Results and Discussion}

No clinical manifestations of the intoxication syndrome were significantly higher in the I group (78.6\% vs. $13.3 \%, \chi^{2}=74.9 ; P<0.001$ ) in comparison with the II group and manifestation intoxication syndrome in the II group were significantly higher in comparison with the I group (61.5\% vs. $4.3 \%, \chi^{2}=53.1$; $P<0.001$ ) (Table 1).

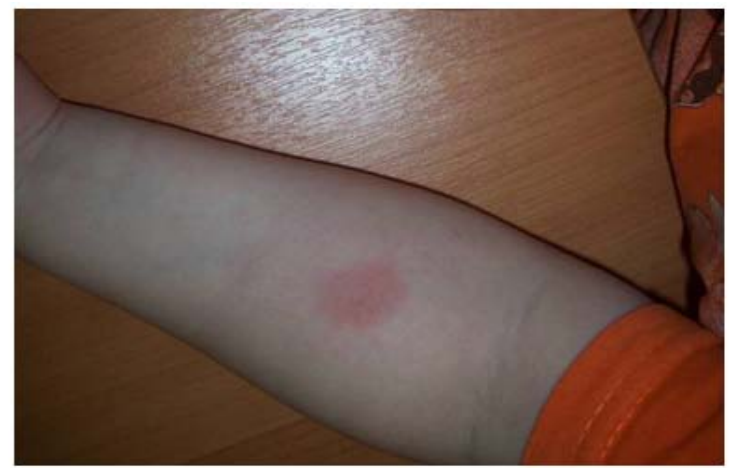

(b) Diaskintest $\mathrm{B}-\mathrm{p} 20 \mathrm{~mm}$ 
Table 1 Clinical manifestations of the intoxication syndrome in the groups.

\begin{tabular}{|c|c|c|c|}
\hline \multirow{2}{*}{ Groups } & No intoxication syndrome & Moderate intoxication syndrome & Manifestation intoxication syndrome \\
\hline & $(\%, n)$ & & \\
\hline I group $(n=70)$ & $78.6 \%(55)^{*}$ & $17.1 \%(12)$ & $4.3 \%(3)$ \\
\hline II group $(n=143)$ & $13.3 \%(19)$ & $25.2 \%(36)$ & $61.5 \%(88) *$ \\
\hline
\end{tabular}

$* P<0.001$.

In Fig. 3, in the II group high sensitivity to TST was observed in $42 \%$ cases, which was significantly higher in comparison with the control group (42.0\% vs. $20.0 \%$, $\left.\chi^{2}=9.99, P<0.01\right)$. Results of DST and QFT tests were significantly higher in the II group compared to I group. Results of DST and results of QFT were comparable in 95\% cases.

Positive DST was marked in $84.4 \%$ in the II group, which was significantly higher compared to the I group (41.5\%, $\left.\chi^{2}=40.36, P<0.001\right)$. At the same time, negative DST was detected significantly more frequently among healthy children in the group I (50.7\% vs. $12.7 \%, \chi^{2}=52.16, P<0.001$ ).

Negative QFT test result in the group I (68.1\%) was significantly higher negative results in the II group (23.1\%), as well positive results of QFT test have significant difference between groups: (30\% in the I group) vs. (76.9\% in the II group).

Analysis of leukocyte subsets being compared to normal ranges revealed significant decrease in $\mathrm{CD}^{+}$

Tuberculin skin test

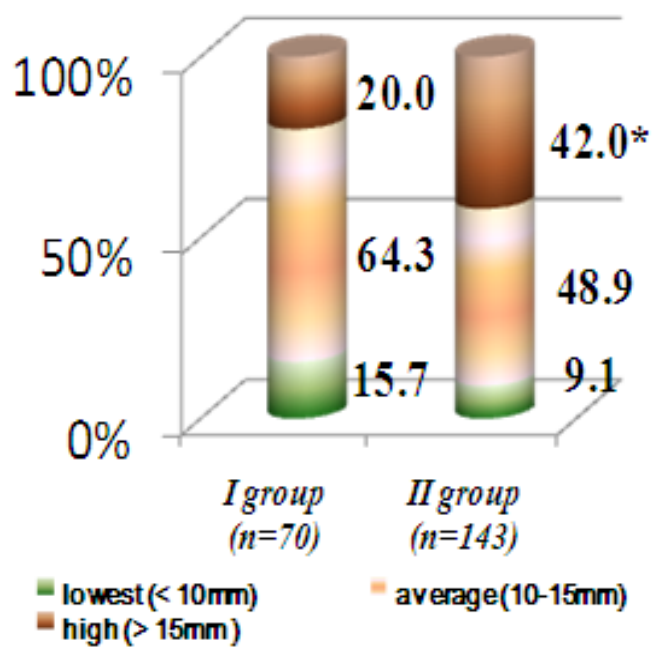

(57.1 vs. 37.7, $\chi^{2}=3.99, P<0.05$ ) and twice lower the level of $\mathrm{CD}^{+} 5^{+}\left(11.9\right.$ vs. 26.4, $\left.\chi^{2}=4.68, P<0.05\right)$ in children infected by MBT may be considered as a sign of immunosuppression (Fig. 4). In the II group (in children with tuberculosis of intrathoracic lymph nodes), the increased level of $\mathrm{CD}^{+}$(14.2 vs. $4.8, \chi^{2}=$ 38.74, $P<0.01$ ) and $\mathrm{CD}_{16} 6^{+}$(58.5 vs. 2.4, $\chi^{2}=38.74$, $P<0.001$ ) may be assumed as a sign of activation. In both groups, elevation of $\mathrm{CD} 25^{+}$and $\mathrm{CD} 95^{+}$levels were observed with the same frequency of occurrence.

No significant differences were identified between groups in the levels of induced cytokines, nevertheless the tendency for elevation of TNF- $\alpha$ and IL-4 in children with TB of intrathoracic lymph nodes was marked (II group) (Fig. 5).

Comparison of the levels of specific immunoglobulins between groups revealed significant elevation of IgM ( $45.8 \%$ vs. $25.6 \%, \chi^{2}=4.07, P<0.05$ ) and a tendency with high level of IgG in children with TB of intrathoracic lymph nodes (Fig. 6).

\section{Diaskintest QuantiFERON-TB}

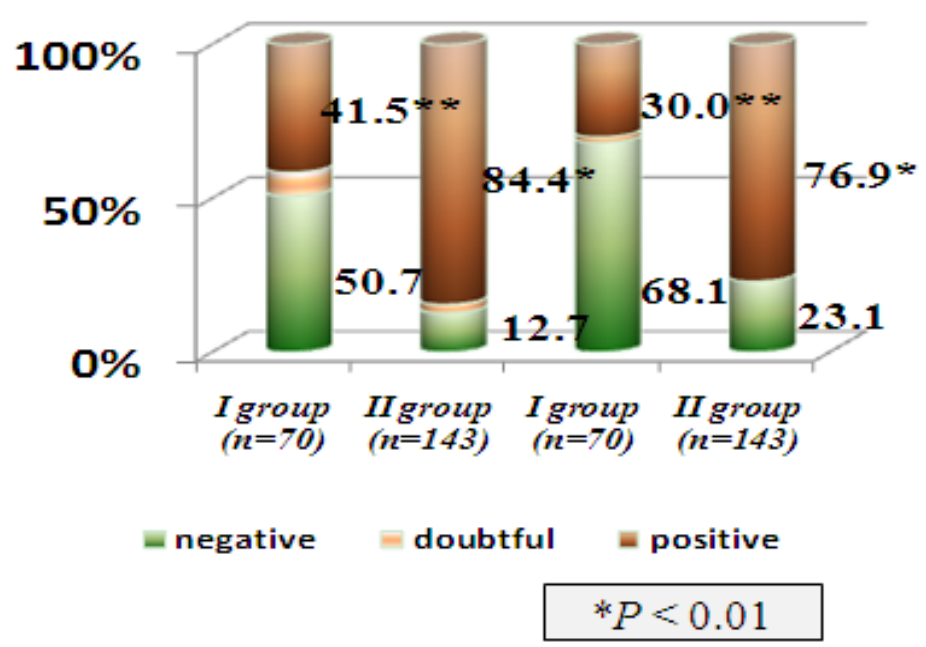

Fig. 3 Results of TST, DST and QFT. 


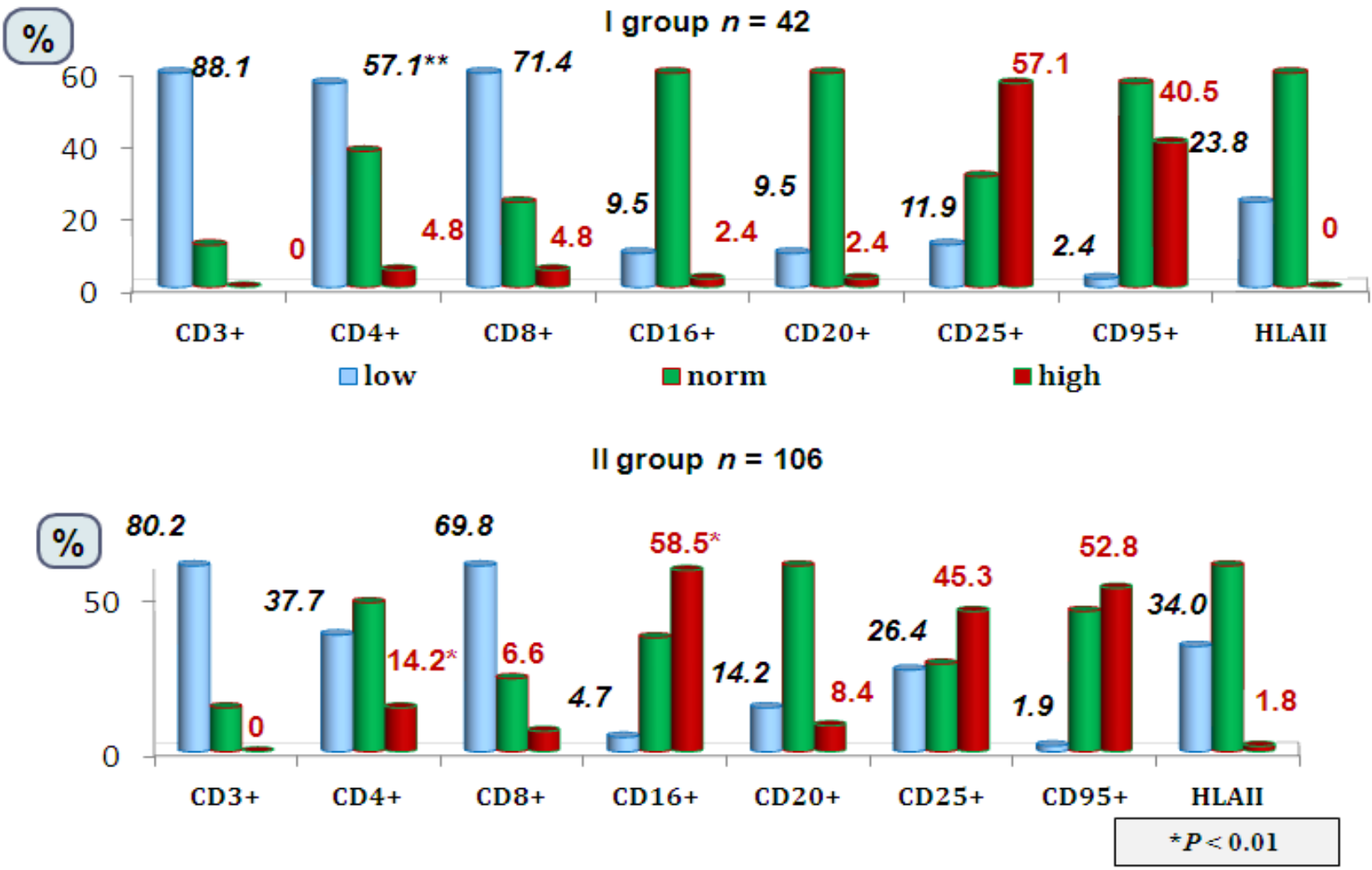

Fig. 4 Leucocytes' subsets in the groups.
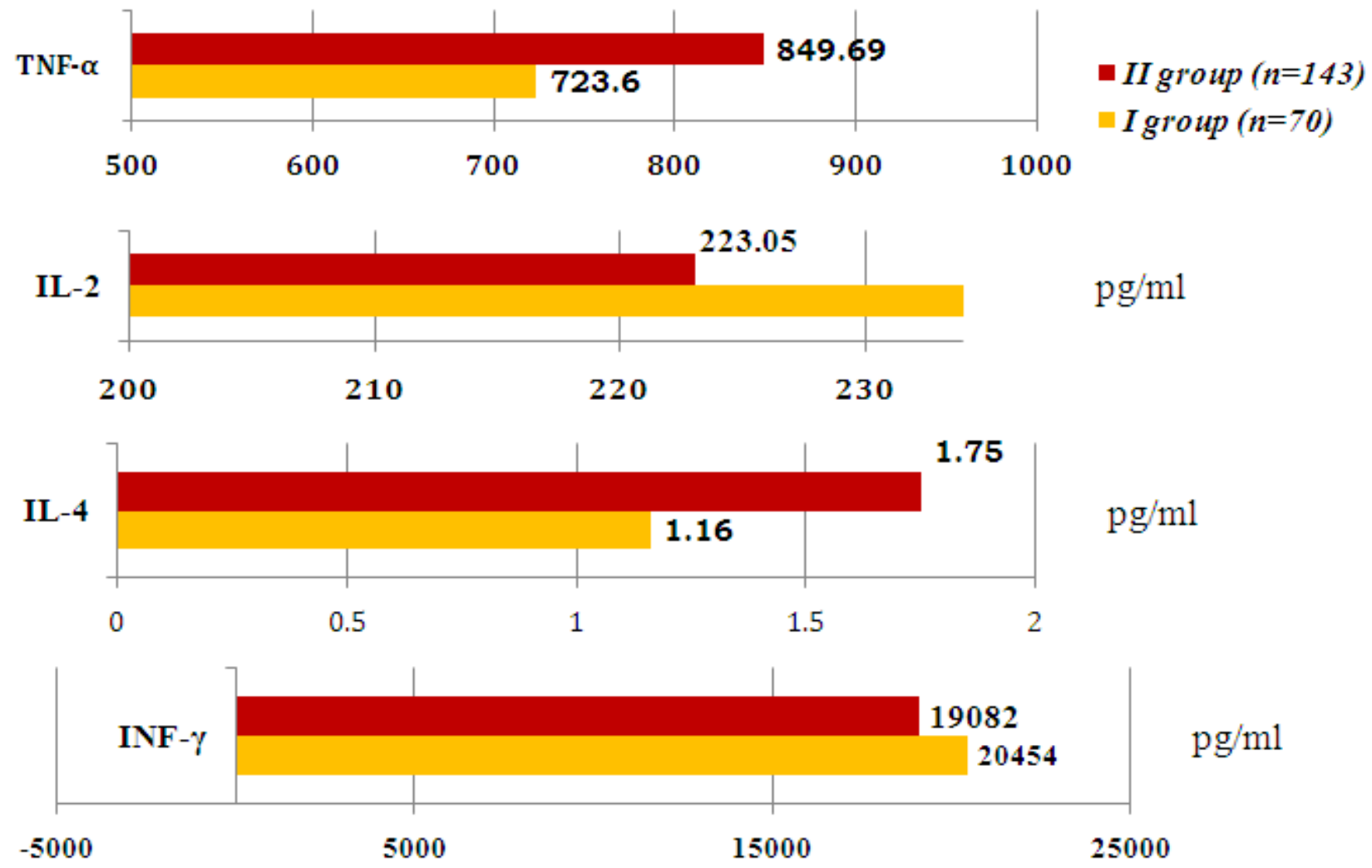

Fig. 5 Levels of cytokine-induced. 


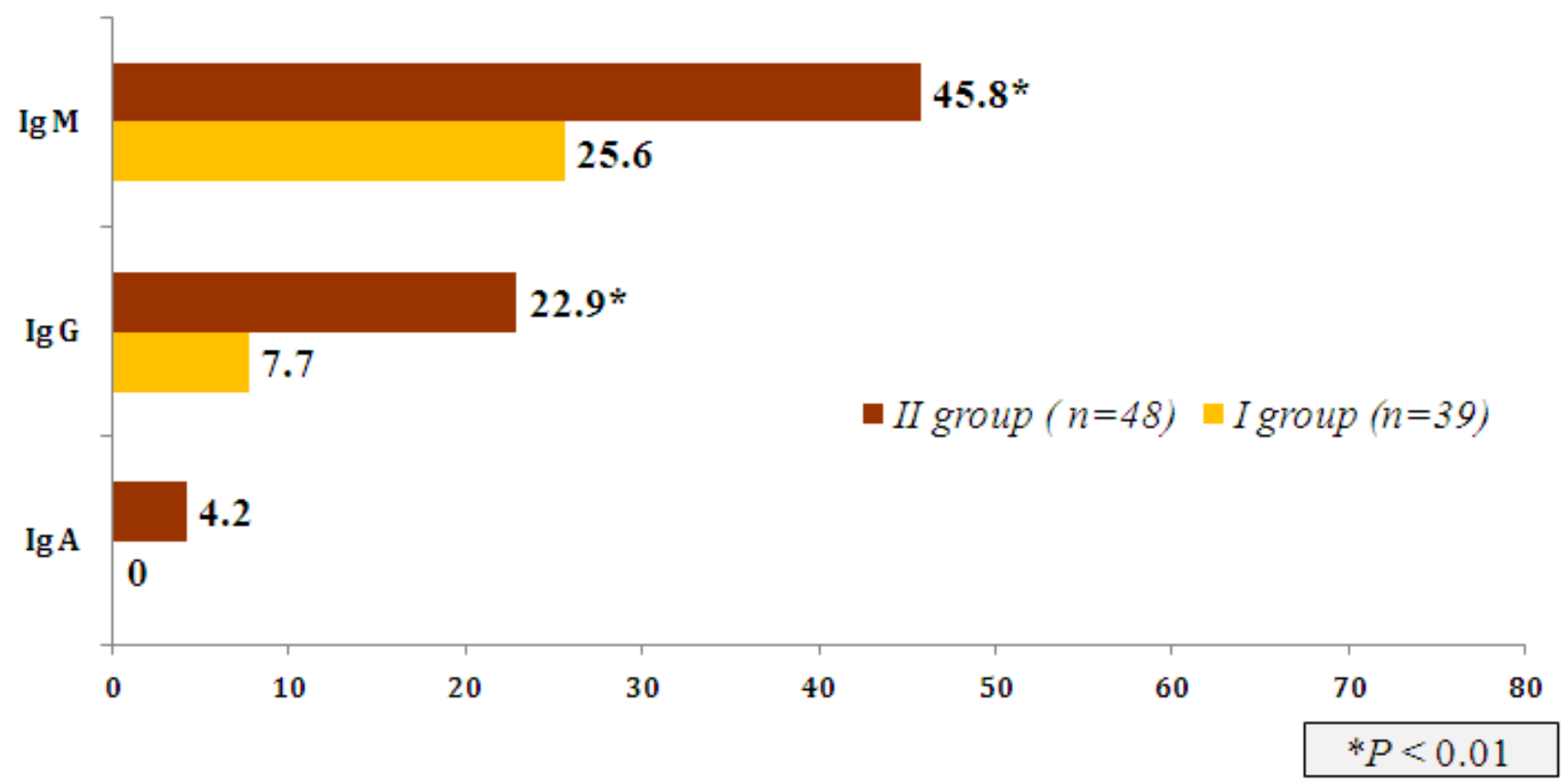

Fig. 6 Levels of specific antibodies in immunological reaction.

Collected in the research data allowed us to figure out diagnostic values of the most relevant immunologic methods. The calculated data on the diagnostic value of TTS (DSS-91.5\%, DSC-15.7\%, PPV-74.4\%, NPV - 45.8\%, and DE-53.6\%) gives evidence of its low degree of information and the pressing need for the introduction of new methods to determine tuberculosis activity. New immunologic methods have higher specificity and diagnostic value in comparison with TT that makes their implementation in the diagnosis of TB in children essential. The data of the diagnostic value of DST (DSS-84.3\%, DSC-59.1\%, PPV-61.9\%, NPV-82.7\%, DE-71.7\%) and the QFT (DSS-78.4\%, DSC-68.2\%, PPV-57.7\%, NPV-85.1\%, DE-73.3\%) do not exhibit significant differences among themselves. However, the degree of information provided by DST is twice as high as the data from TTS, which are confirmed during QFT.

Thus, TTS does not provide sufficient information to determine the activity of tuberculosis infection in children infected with MBT, which leads to a late diagnosis of the disease and identification of the specific process involved in the phase of reverse development.

\section{Conclusions}

In children with tuberculosis, no significant immunologic differences vs. normal ranges were identified, except elevated rate of $\mathrm{CD}_{16}{ }^{+}$. The most informative signs of active tuberculosis in children are positive QFT-G, DST and higher levels of specific IgM. QFT-G and DST are very informative tests in diagnosis of tuberculosis in children and these tests are comparable.

\section{References}

[1] Tuberculosis in Russia in 2010, Analytical Review in Russian Federation, Moscow, Russian Federation, 2011. (in Russian)

[2] Tuberculosis in Russia in 2011, Analytical Review in Russian Federation, Moscow, Russian Federation, 2013. (in Russian)

[3] E.L. Cuevas, R. Browning, P. Bossuyt, M. Casenghi, M. F. Cotton, A.T. Cruz, et al., Evaluation of tuberculosis diagnostics in children: Two methodological issues for conducting and reporting research evaluations of tuberculosis diagnostics for intrathoracic tuberculosis in children, Consensus from an expert panel, Journal Infect. Disease 205 (Suppl. 2) (2012) 209-215.

[4] E.S. Ovsyankina, Clinical and radiological characteristics of new-onset tuberculosis of intrathoracic lymph nodes in children, Problems of Tuberculosis and Lung Disease 1 
(2007) 3-5. (in Russian)

[5] E.S. Ovsyankina, Experience with the new skin test (Diaskintest $\left.{ }^{\circledR}\right)$ for the diagnosis of pulmonary tuberculosis in children and adolescents in the tuberculosis department, Problems of Tuberculosis and Lung Disease 1 (2009) 16-19. (in Russian)

[6] L.V. Slogotskaya, Ya.A. Kochetkov, O.Yu. Senchihina, Using Diaskintest in examining social contact with TB patients among adolescents, Tuberculosis and Lung Disease 5 (2011) 163. (in Russian)

[7] I.F. Dovgalyuk, N.V. Korneva. Clinical and epidemiological features of tuberculosis in children of north-west of the Russian Federation, Tuberculosis and Lung Disease 3 (2011) 12-16. (in Russian)

[8] M. Orme, The latent tuberculosis bacillus (I will let you know if I ever meet one), Int. J. Tuberculosis and Lung Disease 5 (7) (2001) 589-593.

[9] I.E. Tyurin, Computed Tomography of the Chest, ELBI-SPB, St. Petersburg, 2003. (in Russian)

[10] Ya.V. Lazareva, The value of computed tomography in the diagnosis and classification of tuberculosis, Problems of Tuberculosis and Lung Disease, 12 (2005) 14-19. (in Russian)

[11] F.E. Gegeeva, Clinical and radiographic diagnosis of "minor" forms of tuberculosis of intrathoracic lymph nodes in children, Ph.D. Thesis, Central Research Institute of Tuberculosis, Moscow, Russian Federation, 2006. (in Russian)

[12] Y.A. Dauletova, Optimization of the diagnosis of tuberculosis of intrathoracic lymph nodes in children, Ph.D. Thesis, Novosibirsk Institute of Tuberculosis Novosibirsk, Russian Federation, 2009. (in Russian)

[13] V.A. Aksenova, Diaskintest in assessing the activity of tuberculosis in children and adolescents, Tuberculosis and Lung Disease 10 (2009) 13-16.

[14] B.L. Mednikov, L.V. Slogotskaya, Skin test with the drug Diaskintest ${ }^{\circledR}$ (recombinant allergen TB $0.2 \mathrm{mg}$ in $0.1 \mathrm{ml}$ solution for intradermal injection) for the identification of tuberculosis infection, A Handbook for Physicians, ZAO "Masterfarm”, Moscow, Russian Federation, 2009. (in Russian) 